\title{
Synthesis and Characterization of Some Fluorinated 1, 5 - Benzothiazepines and Pyrazolines
}

\author{
S. G. JAGADHANI*, S. G. KUNDLIKAR and B. K. KARALE \\ Department of Chemistry, Radhabai Kale Mahila Mahavidyalaya, Ahmednagar - 414001 , India. \\ ${ }^{*}$ Corresponding author E-mail: suniljagadhani@gmail.com \\ http://dx.doi.org/10.13005/ojc/310177 \\ (Received: December 12, 2014; Accepted: January 20, 2015)

\begin{abstract}
4-Bromo-2-fluorobenzaldehyde 1 when treated with substituted hydroxy acetophenones 2 yields chalcones 3 . These chalcones were refluxed with 2 -aminothiophenol gave "2-[2-(4bromo-2-fluorophenyl)-2,3-dihydro-1,5-benzothiazepin-4-yl]phenol" 4 and when treated with hydrazine hydrate gave the compound "2-[5-(4-bromo-2-fluorophenyl)-4,5-dihydro-1H-pyrazol3 -yl]phenol" 5 . The structures of compounds have been established on the basis of spectral data.
\end{abstract}

Key words: Fluorinated Chalcones, Benzothiazepines, Pyrazolines.

\section{INTRODUCTION}

One of the most important factors in the drug design is that fluorine is much more lipophilic than hydrogen, so incorporating fluorine in the molecule makes it more fat soluble, so it percolates into the membrane more readily and hence fluorinated molecule has a higher bioavailability. Around fifth of all drugs on the market today contain at least one fluorine atom such as Paroxetine, Ezetimibe, Linezolid and Midazolam. Many fluorinated compounds are widely used as antimalarial, antiviral, antipsychotic and antidepressants. Some heterocyclic compounds also act as dyes, pesticides, luminophores and herbicides in nature ${ }^{1}$. Various biological activities associated with chalcones includes antimitotic ${ }^{2}$, antiinvasive $e^{3,4}$, antifungal ${ }^{5}$, antituberculosis ${ }^{6}$, antileishmanial ${ }^{7}$, anti- malarial $^{8,9}$, antiinflammatory ${ }^{10-12}$, antitumor and antioxidant properties ${ }^{13}$. Their recognized synthetic utility in the preparation of pharmacologically interesting heterocycles as pyrazolines, which includes antiparasitary ${ }^{14}$, anti-tumor ${ }^{15}$, nitric oxide synthase inhibitors ${ }^{16}$ and anti-inflammatory ${ }^{17}$ activities.

Benzothiazepines retained the interest of researchers due to the unique structural properties and broad spectrum of biological activities of the compounds ${ }^{18}$. Three possible benzocondensed derivatives of 1,5-benzothiazepines viz. 1,4-, 4,1and 1,5-benzothiazepines ${ }^{19}$ are kwown. Benzothiazepines have their role in the treatment of muscle relaxant ${ }^{20}$, cardiovascular disorders ${ }^{21}$, as 
$\mathrm{Ca}^{2+}$ channel blockers ${ }^{22}$ and inhibitors of HIVintegrase ${ }^{23}$. Pyrazolines reported to have antiinflammatory ${ }^{24-26}$, anti-viral ${ }^{27}$, anti-cancer ${ }^{28-30}$, antidiabetic $^{31}$ and anti-oxidant ${ }^{32}$ properties. Several pyrazoline derivatives found to possess antimicrobial ${ }^{33}$ and anti-HIV ${ }^{34}$ activities. Some of the pyrazolines were effective in inhibiting the accumulation of prion protein ${ }^{35}$, the abnormal protease-resistant form.

\section{Present work}

Substituted hydroxy acetophenones 2 on reaction with 4-bromo-2-fluorobenzaldehyde 1 stirred at room temperature for $24 \mathrm{hrs}$ gives respective chalcones 3 which on reaction with 2amino thiophenol \& reflux for 8 hrs gave benzothiazepines 4 and with hydrazine hydrate for 6 hrs which gave pyrazolines 5 .

\section{EXPERIMENTAL}

All the recorded melting points were determined in open capillary tubes and are uncorrected. I.R. spectra were recorded on Shimadzu FTIR Spectrophotometer in $\mathrm{KBr}$ disc. ${ }^{1} \mathrm{H}$ NMR spectra were recorded on a Bruker Avance II $400 \mathrm{MHz}$ spectrophotometer DMSO- $\mathrm{d}_{6}$ as a solvent and TMS as an internal standard (chemical shift in $\delta$ values). Mass spectra were obtained on a Finnigan mass spectrometer. Purity of the compounds was checked by TLC on silica gel G plates.

\section{Synthesis of Chalcones}

Compound $2(0.005 \mathrm{~mol}) \& 1(0.005 \mathrm{~mol})$ were taken in $100 \mathrm{ml}$ RBF with $25 \mathrm{ml}$ ethanol. To this reaction $2 \mathrm{gm}$ of $\mathrm{KOH}$ was added \& resulting reaction was stirred at room temperature for $24 \mathrm{hrs}$. Then contents were poured over crushed ice \& acidified with conc. $\mathrm{HCl}$, solid thus obtained were separated by filtration \& crystallized from ethanol to get compound 3 . Their characterization data is in the table-1(3a-3e).

\section{Spectral data}

3a I.R. $\left(\mathrm{KBr}, \mathrm{cm}^{-1}\right)$ : 3059 ( $\left.\mathrm{Ar}=\mathrm{C}-\mathrm{H}\right), 2920$ (C-H ), $1649(-\mathrm{C}=\mathrm{O}), 1581(-\mathrm{C}=\mathrm{C}), 1211$ ( $-\mathrm{C}-\mathrm{F})$, 1022 (-C-Br); NMR (DMSO/ d 6 ): $\delta 2.34-3.36(3 \mathrm{H}, \mathrm{s}$, $\left.\mathrm{CH}_{3}\right), 6.86-8.12(6 \mathrm{H}, \mathrm{m}, \mathrm{Ar} \&=\mathrm{CH}$ protons $), 12.30$ $(1 \mathrm{H}, \mathrm{s},-\mathrm{OH})$.

\section{Synthesis of Benzothiazepines}

Compound 3 was dissolved in minimum quantity of ethanol. To this, 4-6 drops of 2aminothiophenol was added and the resulting reaction was refluxed for $4 \mathrm{hrs}$. Then reaction mixture was acidified by using $2 \mathrm{ml}$ acetic acid and heating was continued for next $4 \mathrm{hrs}$. After cooling pale yellow crystals 4 were obtained. These were filtered<smiles>[R]c1c([R])c(O)c(C2=Nc3ccccc3SC(c3ccc(Br)cc3F)C2)c(C(C)=O)c1[R]</smiles>

Scheme 1: 
Table 1: Characterization data of synthesized compound

\begin{tabular}{lcccccc}
\hline Compound & $\mathbf{R}_{\mathbf{1}}$ & $\mathbf{R}_{\mathbf{2}}$ & $\mathbf{R}_{\mathbf{3}}$ & $\mathbf{R}_{\mathbf{4}}$ & M. P. $\left({ }^{\circ} \mathbf{C}\right)$ & Yield (\%) \\
\hline $3 \mathrm{a}$ & $\mathrm{H}$ & $\mathrm{H}$ & $\mathrm{Br}$ & $\mathrm{H}$ & 174 & 65 \\
$3 \mathrm{~b}$ & $\mathrm{Cl}$ & $\mathrm{H}$ & $\mathrm{Cl}$ & $\mathrm{H}$ & 140 & 72 \\
$3 \mathrm{c}$ & $\mathrm{H}$ & $\mathrm{CH}_{3}$ & $\mathrm{Cl}$ & $\mathrm{H}$ & 130 & 67 \\
$3 \mathrm{~d}$ & $\mathrm{H}$ & $\mathrm{H}$ & $\mathrm{CH}_{3}$ & $\mathrm{H}$ & 110 & 62 \\
$3 \mathrm{e}$ & $\mathrm{H}$ & $\mathrm{Cl}$ & $\mathrm{H}$ & $\mathrm{Cl}$ & 162 & 80 \\
$4 \mathrm{a}$ & $\mathrm{H}$ & $\mathrm{H}$ & $\mathrm{Br}$ & $\mathrm{H}$ & 170 & 61 \\
$4 \mathrm{~b}$ & $\mathrm{Cl}$ & $\mathrm{H}$ & $\mathrm{Cl}$ & $\mathrm{H}$ & 130 & 63 \\
$4 \mathrm{C}$ & $\mathrm{H}$ & $\mathrm{CH}_{3}$ & $\mathrm{Cl}$ & $\mathrm{H}$ & 175 & 64 \\
$4 \mathrm{~d}$ & $\mathrm{H}$ & $\mathrm{H}$ & $\mathrm{CH}_{3}$ & $\mathrm{H}$ & 180 & 62 \\
$4 \mathrm{e}$ & $\mathrm{H}$ & $\mathrm{Cl}$ & $\mathrm{H}$ & $\mathrm{Cl}$ & 175 & 62 \\
$5 \mathrm{a}$ & $\mathrm{H}$ & $\mathrm{H}$ & $\mathrm{Br}$ & $\mathrm{H}$ & 110 & 55 \\
$5 \mathrm{~b}$ & $\mathrm{Cl}$ & $\mathrm{H}$ & $\mathrm{Cl}$ & $\mathrm{H}$ & 190 & 52 \\
$5 \mathrm{c}$ & $\mathrm{H}$ & $\mathrm{CH}_{3}$ & $\mathrm{Cl}$ & $\mathrm{H}$ & 240 & 57 \\
$5 \mathrm{~d}$ & $\mathrm{H}$ & $\mathrm{H}$ & $\mathrm{CH}_{3}$ & $\mathrm{H}$ & 165 & 52 \\
$5 \mathrm{e}$ & $\mathrm{H}$ & $\mathrm{Cl}$ & $\mathrm{H}$ & $\mathrm{Cl}$ & 182 & 51 \\
\hline
\end{tabular}

and purified by recrystallization from ethanol. The products obtained were identified with the help of spectral data. Their characterization data is given in the table $1(4 a-4 e)$.

\section{Spectral data}

$4 b$

I.R. $\left(\mathrm{KBr}, \mathrm{Cm}^{-1}\right)$ :3448 (Ar- O-H), $1598(\mathrm{C}=\mathrm{N})$, 1552 ( $\mathrm{C}=\mathrm{C}), 1207$ (-C-F), 1042 ( $-\mathrm{C}-\mathrm{Br}$ ); NMR $\left(\mathrm{DMSO} / \mathrm{d}_{6}\right): \delta 1.14(1 \mathrm{H}, \mathrm{dd}, \mathrm{C}-\mathrm{H}), 2.99(1 \mathrm{H}, \mathrm{dd}, \mathrm{C}-$ $\mathrm{H}), 3.59(1 \mathrm{H}, \mathrm{dd}, \mathrm{C}-\mathrm{H}), 7.28-7.66(8 \mathrm{H}, \mathrm{m}, \mathrm{Ar}-\mathrm{H})$, $7.90(1 \mathrm{H}, \mathrm{d}, \mathrm{Ar}-\mathrm{H}), 15.50(1 \mathrm{H}, \mathrm{OH})$.

\section{Synthesis of pyrazoline}

Compound 3 was taken in $100 \mathrm{ml}$ RBF with $15 \mathrm{ml}$ alcohol. To this reaction mixture $1 \mathrm{ml}$ hydrazine hydrate was added \& the contents were heated under reflux for $3 \mathrm{hrs}$ and to this $2 \mathrm{ml}$ acetic acid was added \& heating was continued for further 2 hrs. After cooling contents were poured over crushed ice. The solid thus obtained was separated by filtration \& crystallized with alcohol to get compounds 5 . The products obtained were identified with the help of spectral data. Their characterization data is given in the table $1(5 a-5 e)$. Spectral data

\section{$5 a$}

I.R. $\left(\mathrm{KBr}, \mathrm{cm}^{-1}\right) 3350(\mathrm{~N}-\mathrm{H}), 2987$ (Ar- =CH), $1602(-\mathrm{C}=\mathrm{N}), 1573(-\mathrm{C}=\mathrm{C}), 1203$ (C-F) 1047 ( C-Br); NMR(DMSO/ $\left.\mathrm{d}_{6}\right): \delta 3.05(1 \mathrm{H}, \mathrm{dd}, \mathrm{C}-\mathrm{H}), 3.67$ $(1 \mathrm{H}, \mathrm{dd}, \mathrm{C}-\mathrm{H}), 5.07(1 \mathrm{H}, \mathrm{dd}, \mathrm{C}-\mathrm{H}), 7.25-7.46(6 \mathrm{H}, \mathrm{m}$, $\mathrm{Ar}$ and $\mathrm{NH}$ proton), $8.09(1 \mathrm{H}, \mathrm{d}, \mathrm{Ar}-\mathrm{H}) 11.82(1 \mathrm{H}$, $\mathrm{OH})$.

\section{REFERENCES}

1. Komeilizadeh H., Iranian J. Pharma. Research, 2006, 4, 229.

2. Ducki S., Forrest R., Hadfield J. A., Kendall A., Lawrence N. J., Mcgown A. T. and Rennison D. Bioorg. Med. Chem. Lett. 1998, 8, 1051.

3. Parmar V. S., Sharma N. K., Husain M., Watterson A. C., Kumar Samuelson L. A.,
Ashok L. C., Prasad A. K., Kumar A., Jha H. N., Olesen C. E., Stove C. P., Bracke M. E. and Mareel, M. M. Bioorg. Med. Chem. 2003, 11, 913.

4. Mukharjee S., Kumar V., Prasad A. K., Raj H. G., Bracke M. E., Olsen C. E., Jain S. C. and Parmar V. S. Bioorg. Med. Chem. 2001, 9, 337. 
5. Lopez S. N., Castelli M. V., Zacchino S. A., Dominguez J. N., Lobo G., Jaime C. C., Cortes J. C. G., Ribas J. C., Devia C., Ana M. R. and Ricardo D. E. Bioorg. Med. Chem. 2001, 9, 1999.

6. Lin Y. M., Zhou Y., Favin M. T., Zhou L. M., Nie W. and Chen F. C. Bioorg. Med. Chem. 2002, 10, 2795.

7. Nielsen S. F., Christensen S. B., Cruciani G., Kharazmi A. and Liljefors T. J. Med. Chem, 1998, 41, 4819.

8. Li R., Kenyon G. L., Cohen F. E., Chen X., Gong B., Dominguez J. N., Davidson E., Kurzban G., Millar R. E., Nuzum E. O., Rosenthal P. J. and Mckerrow J. H. J. Med. Chem. 1995, 38, 5031.

9. Liu M., Wilairat P. and Go M. L. J. Med. Chem. 2001, 44, 4443.

10. Ko H. H., Tsao L. T., Yu K. L., Liu C. T., Wang J. P. and Lin C. N. Bioorg. Med. Chem. 2003, $11,105$.

11. Matsuda H., Morikawa T., Ando S., Iwao T., and Masayuki Y. Bioorg. Med. Chem. 2003, 11, 1995.

12. Herencia Ferrandiz M. L., Ubeda A., Dominguez J. N., Charris J. E., Lobo G. M. and Alcaraz M. J. Bioorg. Med. Chem. Lett. 1998, 8, 1169.

13. Go M. L., Wu X. and Liu X. L. Curr. Med. Chem. 2005, 12, 483.

14. Bhat A. R., Athar F. and Azam A. Eur. J. Med. Chem. 2009, 44, 926.

15. Johnson M., Younglove B., Lee L., LeBlanc R., Holt H., Hills P., Mackay H., Bown T., Mooberry L. S. and Lee M. Bioorg. Med. Chem. 2007, 17, 5897.

16. Carrion M. D., Luisa C., Lopez L. C., Camacho M. E., Tapias, V., Escames G., Castroviejo D. A., Espinosa A., Gallo M. A. and Entrena, A. Eur. J. Med. Chem. 2008, 43, 2579.

17. Ramana M. V., Billa V. K., Pallela V. R., Murlidhar R. M. R., Boominathan R., Gabriel J. L. and Reddy E. P. Bioorg. Med. Chem.
2008, 16, 3907.

18. Jiaxi X. Mol. Div. 2005, 9, 45.

19. Levai A. J. Het. Chem. 1999, 37, 199.

20. Urbankshi M. J., Chen R. H., Demarest K. T., Gunnet J., Look R., Ericson E., Murray W. V., Rybczynski P. J. and Zhang X. Bioorg. Med. Chem. Lett. 2003, 48, 4031.

21. Nakayama K., Nozawa Y. and Fukuta Y., J. Cardiovasc. Pharmacol. 1994, 23, 731.

22. Tarabova B., Lacinova L. and Engel J. Eur. J. Pharmcol. 2007, 753, 39.

23. Santo R. D. and Costi R. I/ Farmaco 2005, 60, 385.

24. Sondhi S. M., Kumar S., Kumar N. and Roy P. Med. Chem. Res. 2011, 1.

25. Reddy M. V., Billa V. K., Pallela V. R., Mallireddigari M. R., Boominathan R., Gabriel J. L. and Reddy E. P., Bioorg. Med. Chem. Lett. 2008, 16, 3907.

26. Sivakumar P. M., Prabhu Seenivasan S., Kumar V. and Doble M., Bioorg. Med. Chem. Lett. 2010, 20, 3169.

27. Bandgar B. P., Gawande S. S., Bodade R. G., Gawande N. M. and Khobragade C. N. Bioorg. Med. Chem. 2009, 17, 8168.

28. Nassar E. J. Am. Sci. 2010, 6.

29. Al-Saadi Saudi M. S. M. Pharm. J. 2008, 16,135.

30. Ahasan N. B. and Islam M. R. Bang. J. Phar. 2008, 2, 81.

31. Rahman M. A. and Siddiqui A. A. Int. J. Pharm. Sci. Drug Res. 2010, 2.

32. Bandgar B. P., Gawande S. S., Bodade R. G., Gawande N. M. and Khobragade C. N., Bioorg. Med. Chem. 2009, 17, 8168.

33. Halnor V. B., Joshi N. S., Karale B. K. and Gill C. H., Indian J. Het. Chem.., 2005, 14, 371.

34. Waheed V. and Khan S. A. Indian J. Het. Chem., 2002, 11, 59.

35. Kimata A., Nakagawa H., Ohyama R., Fukuuchi T., Ohta T. Suzuki S. and Miyata N. J. Med. Chem. 2007, 50, 5053. 